\title{
PERFORMANZ
}

\section{Mit Fragebogen führen}

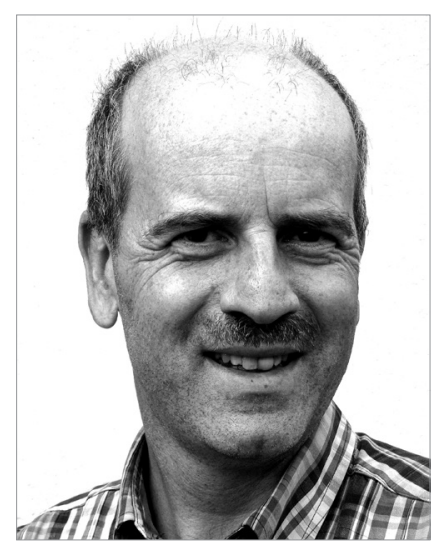

VON ARMIN SCHNEIDER

Prof. Dr. Armin Schneider lehrt und forscht an der Hochschule Koblenz zur empirischen Sozialforschung, zu sozialen und nachhaltigen Dimensionen von Management und Leadership. Er ist Sprecher der Sektion Forschung der Deutschen Gesellschaft für Soziale Arbeit. schneider@hs-koblenz.de

\author{
Die Forderung nach handfesten Nachweisen über die \\ Wirkungen Sozialer Arbeit und sozialer Dienstleistungen \\ können schriftliche Befragungen hilfreich sein.
}

\begin{abstract}
»Wer fragt, der führt «, so ein oft zitiertes Sprichwort. Fragebogen versuchen auf verschiedenen Ebenen unser Verhalten, unsere Einstellungen und unsere Absichten zu ergründen und daraus Schlüsse zu ziehen.

Im Alltag von Sozialunternehmen sind Mitarbeiterbefragungen, Evaluationsfragebogen, Zufriedenheitsbefragungen von Kunden und Klienten nicht mehr wegzudenken. Doch angesichts zu hoher Erwartungen an die Ergebnisse einer Fragebogenerhebung ist Skepsis angebracht.

Damit eine Fragebogenerhebung ihre Wirkung entfalten kann und Führungskräften bei ihrer Aufgabe hilfreich sein kann, sind grundlegende Kenntnisse erforderlich. Die vermeintliche einfache Durchführbarkeit, unterstützt durch zahlreiche einfach zu bedienende Softwareprogramme kann bei Nichtbeachtung zu falschen Sicherheiten verführen.
\end{abstract}

\section{Immer eine Intervention}

Fragebogen sind nie neutral, zeigen Sie doch zumindest ein Erkenntnisinteresse oder ein vermeintliches (anderes) Interesse seines Absenders. Ob Führungskräfte oder externe Institute einen Fragebogen herausgeben, ist dabei zunächst einmal unerheblich.

Allein die Existenz eines Fragebogens führt zu Interpretationen: Was wollen die wissen? Was wollen die mit dem Fragebogen erreichen? Und: Selbst noch so kniffelig ausgedachte Fragebogen mit Querfragen und Kontrollfragen können nicht davon hinwegtäuschen, dass die Antworten immer auch eine "Redaktion" durchlaufen, entweder dergestalt, dass (vermutete) sozial erwünschte Antworten gegeben werden oder die Antworten mit einer Absicht gegeben werden, das eine oder andere zu erreichen oder zu verhindern.

Fragebogen sind zeit-, orts- und situationsgebunden und deren Aussagen sind immer nur in einem Kontext zu verstehen. Eine derzeit geäußerte hohe Zufriedenheit mit dem Management kann in einigen Monaten unter anderen Bedingungen geringer ausfallen. Gerade in Veränderungsprozessen können sogar objektive Verbesserungen negativ werden, weil sich beispielsweise Referenzrahmen bei den Befragten ändern.

Welche Faktoren auch immer auf eine Fragebogenerhebung einwirken: Sobald ein Fragebogen verwendet wird, verhalten sich Personen zu einem Fragebogen, der eine (selten berechenbare) Wirkung entfaltet. Allein eine Fragebogenerhebung kann schon die Wirklichkeit verändern - ohne dass bereits eine Auswertung vorliegt.

Wird in einer Mitarbeiterbefragung nach Veränderungsperspektiven gefragt kann dies je nach Situation Befürchtungen nach Veränderungen hervorrufen oder aber auch überzogene Erwartungen an die eigene Karriere. Wird nach Motivationsfaktoren gefragt, kann hierbei die Angst vor Gehaltskürzungen ebenso ein Motiv für eine Antwort sein wie die Suche nach Anerkennung.

Auf jeden Fall wird das Führungsverhalten nach einer Befragung der Mitarbeiter bewertet: $\mathrm{Ob}$ und wie die $\mathrm{Er}$ gebnisse einer Befragung veröffentlicht werden und welche Schlussfolgerungen wer wie zieht und was konkret als Folge oder in einer (auch nur gefühlten) Verbindung zur Fragebogenerhebung wahrgenommen wird, hat eine Bedeutung und wird auch die Teilnahmebereitschaft an zukünftigen Befragungen erhöhen oder vermindern. 
Diese Anmerkungen zeigen, dass das Forschungsinstrument »Fragebogen « auch ein Interventions- und Diagnoseinstrument ist, sowohl auf individueller als auch auf betrieblicher oder organisatorischer Ebene. Daher gilt es seinen Nutzen im Vorfeld gut abzuwägen. Einsatzmöglichkeiten von Fragebogen in sozialen Diensten und Einrichtungen können beispielsweise sein:

- Erkenntnisgewinnung und Forschung: Befragung von Mitarbeitern zur Bewertung von Personalentwicklungsmaßnahmen, Zufriedenheit von Heimbewohnern, Bedarfserhebung für Dienste in einem Stadtteil

- Diagnose: Erhebung von Ressourcen und Problemlagen von Klienten der Jugendhilfe, Einschätzung von Gefährdungen beispielsweise im Kontext von Kinderschutz, Analyse der Mitarbeiterbindung in einzelnen Arbeitsfeldern

- Intervention: aktivierende Befragung in einem Stadtteil mit besonderem Entwicklungsbedarf, zirkuläre Befragung zur Ressourcenanalyse bei Klienten, Befragung im Rahmen einer Zukunftswerkstatt eines Wohlfahrtsverbandes (vgl. Schneider 2013)

Die ersten Fragen in einer Checkliste vor einer Fragebogenerhebung sind die, ob die Fragestellung klar ist und die damit zu gewinnenden Erkenntnisse auch durch andere Instrumentarien oder bereits vorhandene Daten, gewonnen werden können.

Ebenfalls ist zu fragen, ob und welche Auswirkungen die Fragebogenerhebung haben kann, ob sie für die Zielgruppe geeignet ist und es einen Zugang dazu gibt. Nicht zuletzt bedarf die Ressourcenfrage einer Antwort. Eine Fragebogenerhebung per Hand und Excel auszuwerten ist nicht mehr zeitgemäß und beansprucht mehr Ressourcen als etwa die Auswertung durch ein professionelles Programm, das Fragebogen einscannt und entsprechend auswertet. Auch ist zu fragen, ob eine online-basierte Befragung zu Einsatz kommen kann.

Generell kann ein Fragebogen als eine quantitative Erhebungsmethode sehr schnell zahlenbasierte Informationen liefern, die eine Vergleichbarkeit liefern und eine Orientierung bieten können. Wie alle zahlenbasierten Systeme so wird auch mit einer statistischen
Auswertung die Komplexität auf einige Kennzahlen oder Kennziffern reduziert, die Hinweise auf Steuerungsnotwendigkeiten geben können, als Entscheidungsgrundlage dienen können, - aber nicht müssen. Denn ein Fragebogen kann nicht komplexe Verstehenszusammenhänge erheben und keine umfassende Partizipation oder Kommunikation bieten. Sollten letztere Interessen wichtig sein, so sind statt eines Fragebogens qualitative Methode wie Gruppendiskussionen oder Interviews vorzuziehen oder aber der Fragebogen sollte mittels eines triangulativen Verfahrens mit solchen Methoden verknüpft werden.

Sind alle diese grundsätzlichen und organisatorischen Fragen geklärt, kann die »Operationalisierung " erfolgen. Dazu ist es erforderlich sowohl die Theorie des Befragungsgegenstandes als auch der Fragebogenerhebung zu kennen und zu nutzen. Damit ist nicht gemeint, ein umfassendes Studium vorzunehmen, sondern die wesentlichen bereits vorhandenen Erkenntnisse zu kennen, um die richtigen Fragen zu stellen (Gegenstandsbezogenheit) und die Fragen richtig (Erhebungstechnik) zu stellen.

Die richtigen Fragen ergeben sich aus der Befragungsabsicht, den zuvor formulierten Annahmen (Hypothesen) und der bisherigen Erkenntnissen zum Forschungsgegenstand (auch um Vergleiche herstellen zu können, ob die in der eigenen Organisation gefundenen Ergebnisse "normal « sind oder in besonderer Weise abweichen. Zur Erhebungstechnik gilt es klare, einfache und für alle befragten Personen in gleicher Art verständliche Fragen zu stellen und möglichst Verzerrungen zu vermeiden. Solche Verzerrungen können durch die Reihenfolge der Fragen, durch soziale und kommunikative Phänomene (u. a.) Erwünschtheit, Tendenz zur positiven Selbstdarstellung), psychologische Effekte (Motivation, Reize, Raten etc.), Effekte durch die Befragung und Anlage der Befragung (Verstellung, Nichtbeantwortung, Tendenz zur ersten passenden Kategorie, Tendenz zur mittleren Antwort etc.) entstehen (vgl. Schneider 2013, 139 ff.)

Schließlich darf eine Auswertung nicht bei einer reinen Beschreibung von Phänomenen stehenbleiben (deskriptive Statistik), sondern muss auch Aussagen über eine Generalisierung (Inferenzstatistik) liefern können (vgl. Lehmann 2013, 77). Dazu gehört zumindest die
Aussage, ob und mit welcher Wahrscheinlichkeit die Ergebnisse rein zufällig sind (Signifikanztests) ober ob es (zumindest statistische) Abhängigkeiten zwischen verschiedenen Variablen (und damit auch Merkmalen) gibt (Korrelationen) und inwiefern diese sich unter Zuhilfenahme von Theorie erklären lassen (um nicht auf etwaige Scheinkorrelationen oder (falsch) monokausalen Zusammenhängen »hereinzufallen«.

\section{Warnung zum Schluss}

Gerade in der zunehmenden Diskussion um Wirkungsorientierung und Evidenzbasierung Sozialer Arbeit und sozialer Dienstleistungen ist ein fundiertes Wissen über die Grenzen und Möglichkeiten von Befragungen von großer Bedeutung für die Zukunft sozialer Unternehmen. In dem Maße, in dem Messungen, Daten, Zahlen und Fakten für die Steuerung und Führung von Unternehmen an Bedeutungszuwachs gewinnen, muss auch deren Begrenztheit gesehen werden, zum einen in dem Sinne, dass Landkarten keine Landschaften sind und Zahlen demnach nur (verkürzte) Abbilder der Wirklichkeit und zum anderen in dem Sinne, dass Kreativität in Einrichtung oft nur dort möglich ist, wo rechenschaftsfreie Räume (vgl. Sprenger 2012) bewusst geschaffen und freigehalten werden.

Die Chancen von Fragebögen liegen darin, dass entsprechende fachlich fundierte Indikatoren (wahrnehmbare Eigenschaften) auch komplexer Phänomene beschrieben werden sowie durch entsprechende Kennzahlensysteme abgebildet und kommunikationsfähig gemacht werden können. Um beim Beispiel der Landkarte zu bleiben: Sie kann Orientierung geben, Maßstäbe aufzeigen und Vergleiche ermöglichen, aber das nur in wenigen abgezählten Dimensionen.

\section{Literatur}

Faulbaum, Frank, Prüfer, Peter und Rexroht, Margrit (2009). Was ist eine gute Frage? Wies baden: VS-Verlag.

Lehmann, Robert (2013). Warum die Sozialarbeitsforschung quantitative Methoden entwickeln muss. In: Neue Praxis 1/2013. S. 73-78. Schneider, Armin (2013). Fragebogen in der Sozialen Arbeit. Opladen: Barbara Budrich. Sprenger, Reinhard (2012). Radikal führen. Frankfurt am Main: Campus-Verlag. 